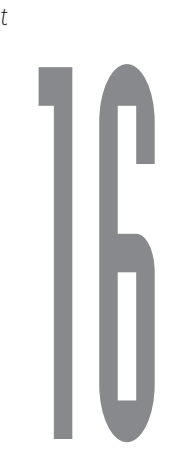

\title{
VALUATION OF PATENTS AND R\&D PROJECTS USING REAL OPTIONS:A PRACTICAL IMPLEMENTATION
}

\author{
LUCÍA ÁLVAREZ \\ FELIPE BLANCO \\ FELIPE RUIZ \\ PABLO SOLANA

\begin{abstract}
DEPARTAMENTO DE INGENIERÍA DE ORGANIZACIÓN, ADMINISTRACIÓN DE EMPRESASY ESTADÍSTICA
ESCUELATÉCNICA SUPERIOR DE INGENIEROS INDUSTRIALES

UNIVERSIDAD POLITÉCNICA DE MADRID
\end{abstract}

\begin{abstract}
Resumen: The correct valuation of an R\&D project is a rather complex process. Aside from the fact that it can take several years to launch a new product after extensive investment, there happens to be a significant abandonment probability at each of the development and exploitation stages. Significant uncertainty is also inherently attached to both development costs and subsequent operational cash flows (be it the case of receiving final approval for commercial purposes). These cash flows need to be estimated with anticipation in order to give a reasonable value for the patent or $R \& D$ project. The approach undertaken herein is to consider the patent or $R \& D$ project as a complex option on relevant underlying stochastic variables: investment costs and operational cash flows. A model is proposed, programmed and applied to a real case.
\end{abstract}

Key words: Real options, Monte Carlo, patents.

\section{Introduction}

Literature on financial option theory applied to valuation of projects (real options methodology) is relatively abundant [see, for instance, Amram et al. (1999) and Copeland et al. (2000)]. However, when the underlying to be valued depends upon more than two stochastic variables, or the process of the underlying is not standard -i.e. geometric Brownian motion, both the formulation and the implementa- tion of the model can become cumbersome. Deep understanding of the dynamics of the project to be valued is needed, as well as a powerful mathematical tool that enables the model to give accurate solutions for a broad range of input values.

This paper poses a model for the valuation of patents and R\&D projects. The uncertainty associated to both investment costs and future cash flows in these types of investments enables the manager of the company to actually exercise a number of options along the life of the project. This is a typical feature that fully justifies the use of the real options framework.

The model presented in this paper roots in a general framework proposed by Schwartz et al. (2004) but deepens further into peculiarities of real investment processes. These conceptual improvements can be summarized as follows:

- A first investment stage is introduced, defined by its deterministic nature, with fixed estimated total cost and a maximum yearly investment rate. This consideration is made upon fairly common budgeting decisions found in the real world when the first stage of a project of this nature is undertaken.

- An abandonment option is also introduced at later stages, once the project has got final approval 
and the product is being sold in the (patent protected) market. Then, at each moment in time, the patent or project can be sold for an exercise value that is modelled as a decreasing function of time down to a null value when the patent expires.

A Monte Carlo approach to solve the model equations is proposed. More precisely, an american type Monte Carlo method for complex options valuation is programmed, taking account of the stochastic nature of both investment costs and cash flows, but also the stopping time nature of the «time to completion» variable within the investment stages.

Full account is also given on the differences with other standard valuation techniques such as NPV.

\section{The model}

Figure I shows the different stages used as a basis for the model.

The first stage is one of deterministic nature. It is characterized by an estimated total investment and a maximum yearly investment rate. It represents the research phase of the project previous to the development of it. At the end of it, a patent is obtained so that it will protect the industrial property during the $T$ following years. An abandonment option is considered while at this stage. In this paper its length is noted by $T^{\prime}{ }^{\prime}$.

The second stage represents the development of the project under the protection of the patent. It is one of stochastic nature and is defined by an estimated total investment, its variance and a maximum yearly investment rate. During this stage the project could be either abandoned or sold. In this paper its expected length is noted by $T_{k}$.

At both the first and second stages, the possibility of a «catastrophic event» that could plunge the project value down to zero, such as the discovery of a pernicious side effect, is taken into account.

The third and final stage is the one at which the cash flows derived from the exploitation of the patent are realized. Here an abandonment/sell option is also considered.

\section{I. Modelling the underlying}

The following controlled diffusion equation is used to describe the process associated to the investment costs:

$$
d K=-I d t+\sigma(\mathbb{K})^{\frac{1}{2}} d z
$$

Where $\mathrm{K}$ is the total cost to completion, I the investment per unit time, $\sigma(I K)^{1 / 2} d z$ the volatility of this process and $\mathrm{dz}$ a Wiener process, uncorrelated with the market.The term represents technical uncertainty.

Solving for the variance of the cost process, we find:

$$
\operatorname{Var}(K)=\frac{\sigma^{2} K^{2}}{2-\sigma^{2}}
$$

In order to incorporate the aforementioned possibility of a «catastrophic event» in both the research and development stages, we assume that such events follow a Poisson distribution with a $\lambda$ parameter equal to the expected number of occurrences per time unit considered.

Figure I

Stages of the project

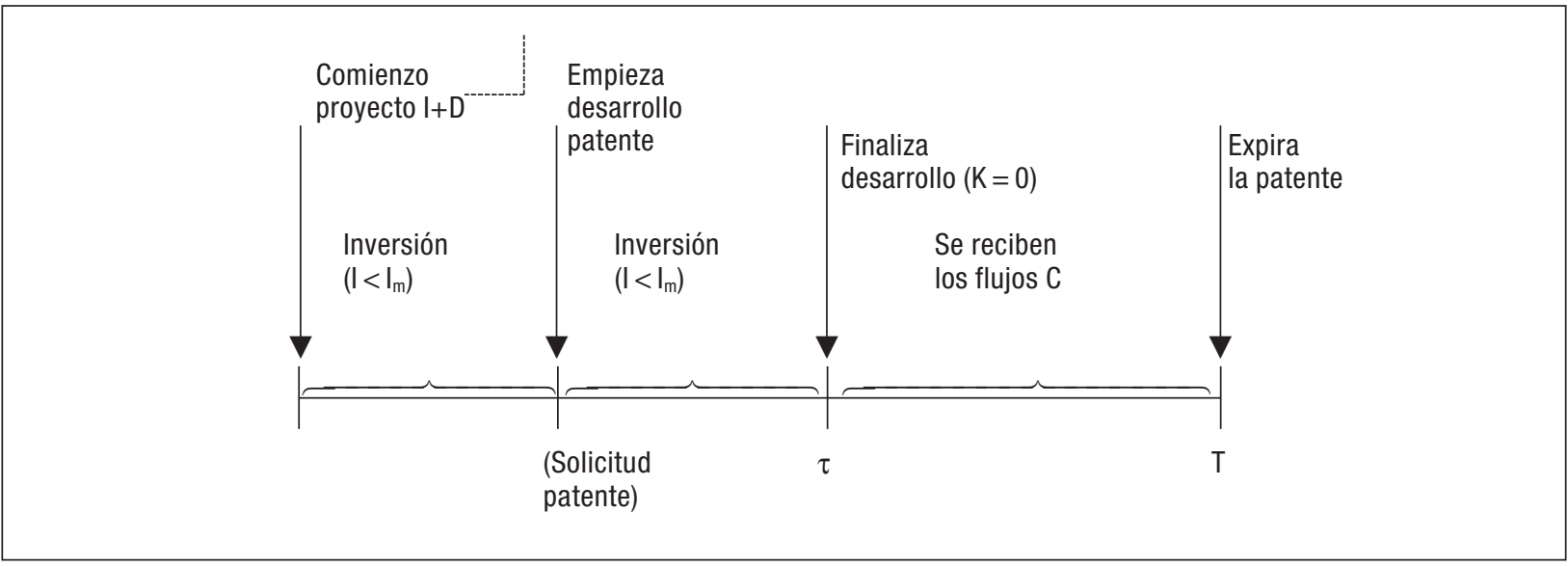




$$
e^{-\lambda T_{K}}=p
$$

Dynamics of the cash flow rate are defined by a standard geometric Brownian motion:

$$
d C=\alpha C d t+\phi C d w
$$

where $d w$ is a Wiener process perfectly correlated with the market portfolio. Cash flows, it should be noted, become real once a full development of the patent is achieved.

\subsection{The value of the project without options}

A «dry» value of the project can be estimated, without options. This step is rather important as it is this dry value the final underlying upon which we built up the real option valuation process.

Let's call $\mathrm{V}(\mathrm{C}, \mathrm{t})$ the value of the project for a given cash flow rate $\mathrm{C}$ at a time t.Thus, the value of the Project must satisfy the following partial differential equation (provided that the residual Project value is some $M$ times the value of the last cash flow considered):

$$
\frac{1}{2} \phi^{2} C^{2} V_{C C}+\alpha^{*} C V_{C}+V_{t}-r_{f} V+C=0
$$

Being $=\alpha^{*}=\alpha-\eta$

With the boundary condition:

$$
V\left(C_{t=T}, T\right)=M \cdot C_{t=T}
$$

For a proof of this, follow Schwartz (2004) but please, note that after solving this EDP and performing the pertinent operations, the $V$ dynamics is not of a Brownian-Geometric nature and as a consequence, the volatility of the project at this stage is not necessarily the same as the one of the underlying cash flow rate.

The solution of the EDP is

$$
\begin{gathered}
N P V=V\left(C_{0}, 0\right)=\frac{C_{0}}{r_{f}-\alpha^{*}}\left[1-e^{-\left(r_{f}-\alpha^{*}\right) \cdot T}\right]+ \\
+M \cdot C_{0} \cdot e^{-\left(r_{f}-\alpha^{*}\right) \cdot T}
\end{gathered}
$$

In this way we know the dry value of the project, except for the value of the parameter $C_{0}$.

When $\delta=0$ the former expression simplifies to:

$$
N P V=C_{0} \cdot(T+M)
$$

$C_{0}$ and $\alpha$ represent the cash flow rate at the beginning of the project and its real yield respectively. These parameters are estimated this way:

A least square adjustment is performed in order to fit the expected annual cash flows at the exploitation stage to the expression $C_{t}=a \cdot e^{b \cdot t}$. The b parameter will be our $\alpha$ estimate.

Then, we calculate the standard NPV and put this result into the analytic expressions of the value of the project without volatility and options. In these expressions the only unknown parameter would be $C_{0}$.

As an extension of the previous work we consider the following:

\section{Existence of a development stage}

Let be $F(C, K, t)$ the value of the project during the patent development investment stage. It should satisfy the following PDE.

$$
\begin{gathered}
\frac{1}{2} \phi^{2} C^{2} F_{C C}+\frac{1}{2} \sigma^{2} I K F_{K K}+\phi \sigma \rho C(I K)^{\frac{1}{2}} F_{C K}+ \\
+\alpha^{*} C F_{C}-I F_{K}+F_{t}-\left(r_{f}+\lambda\right) F-1=0
\end{gathered}
$$

with the boundary condition:

$$
\begin{gathered}
F(C, 0, \tau)=V(C, \tau)=\frac{C}{r_{f}-\alpha^{*}}\left[1-e^{-\left(r_{f}-\alpha^{*}\right)(T-\tau)}\right]+ \\
+M \cdot C \cdot e^{-\left(r_{f}-\alpha^{*}\right)(T-\tau)}
\end{gathered}
$$

$t=\tau$ is the end of investment time step when the cash flows start to be received. Note that $\tau$ is a stochastic variable.

If $\delta \neq 0$ its solution without uncertainty and options would be:

$$
\begin{aligned}
& N P V=V\left(C_{0} e^{\alpha T_{K}}, T_{K}\right) e^{\left.-\left[\left(r_{f}+\lambda\right)+\eta\right)\right] T_{K}}-\int_{0}^{T_{K}} I_{m} e^{-\left(r_{f}+\lambda\right) t} d t= \\
& =V\left(C_{0}, T_{K}\right) e^{-\left[\left(r_{f}+\lambda\right)-(\alpha-\eta)\right] T_{K}}-I_{m}\left[-\frac{e^{-\left(r_{f}+\lambda\right) t}}{r_{f}+\lambda}\right]_{t=0}^{t=T_{K}}= \\
& =V\left(C_{0}, T_{K}\right) e^{-\left[\left(r_{f}+\lambda\right)-(\alpha-\eta)\right] T_{K}}-\frac{I_{m}}{r_{f}+\lambda}\left(1-e^{-\left(r_{f}+\lambda\right) T_{K}}\right)
\end{aligned}
$$


Substituting in the previous expression, the analytic NPV turns out to be:

$$
\begin{aligned}
& N P V=\left(\frac{C_{0}}{r_{f}-\alpha^{*}}\left[1-e^{-\left(r_{f}-\alpha^{*}\right)\left(T-T_{K}^{\prime}\right)}\right]+\right. \\
&\left.+M \cdot C_{0} \cdot e^{-\left(r_{f}-\alpha^{*}\right)\left(T-T_{K}\right)}\right) e^{-\left[\left(r_{f}+\lambda\right)-(\alpha-\eta)\right] T_{K}}- \\
&-\frac{I_{m}}{r_{f}+\lambda}\left(1-e^{-\left(r_{f}+\lambda\right) T_{K}}\right)
\end{aligned}
$$

If $\delta=0$, the preceding expression could be simplified:

$$
N P V=C_{0} \cdot\left(T-T_{K}+M\right) \cdot e^{-\lambda \cdot T_{K}}-\frac{I_{m}}{r_{f}+\lambda}\left(I-e^{-\left(r_{f}+\lambda\right) T_{K}}\right)
$$

Existence of both research and development stages

If $\delta \neq 0$ :

$$
\begin{gathered}
N P V=\left(\frac{C_{0}}{r_{f}-\alpha^{*}}\left[I-e^{-\left(r_{f}-\alpha^{*}\right)\left(T-T_{K}\right)}\right]+\right. \\
\left.+M \cdot C_{0} \cdot e^{-\left(r_{f}-\alpha^{*}\right)\left(T-T_{K}\right)}\right) \cdot \\
\cdot e^{-\left[\left(r_{f}+\lambda\right)-(\alpha-\eta)\right] T_{K}} \cdot e^{-\left[\left(r_{f}+\lambda^{\prime}\right)-(\alpha-\eta)\right] T_{K}^{\prime}}- \\
-\frac{I_{m}}{r_{f}+\lambda} \cdot\left(I-e^{-\left(r_{f}+\lambda\right) T_{K}}\right) \cdot e^{-\left(r_{f}+\lambda^{\prime}\right) T_{K}^{\prime}}-\frac{I_{m}^{\prime}}{r_{f}+\lambda^{\prime}} . \\
\cdot\left(I-e^{-\left(r_{f}+\lambda^{\prime}\right) T_{K}^{\prime}}\right)
\end{gathered}
$$

If $\delta=0$ the prior expression simplifies to:

$$
\begin{gathered}
N P V=C_{0} \cdot\left(T-T_{K}+M\right) \cdot e^{-\lambda \cdot T_{K}} \cdot e^{-\lambda^{\prime} \cdot T_{K}^{\prime}}-\frac{I_{m}}{r_{f}+\lambda} \cdot \\
\cdot\left(I-e^{-\left(r_{f}+\lambda\right) T_{K}}\right) \cdot e^{-\left(r_{f}+\lambda^{\prime}\right) T_{K}^{\prime}}-\frac{I_{m}^{\prime}}{r_{f}+\lambda^{\prime}} \cdot\left(I-e^{-\left(r_{f}+\lambda^{\prime}\right) T_{K}^{\prime}}\right)
\end{gathered}
$$

\subsection{The options}

The options taken into account are summarized in Figure 2:
Figure 2

Options profile

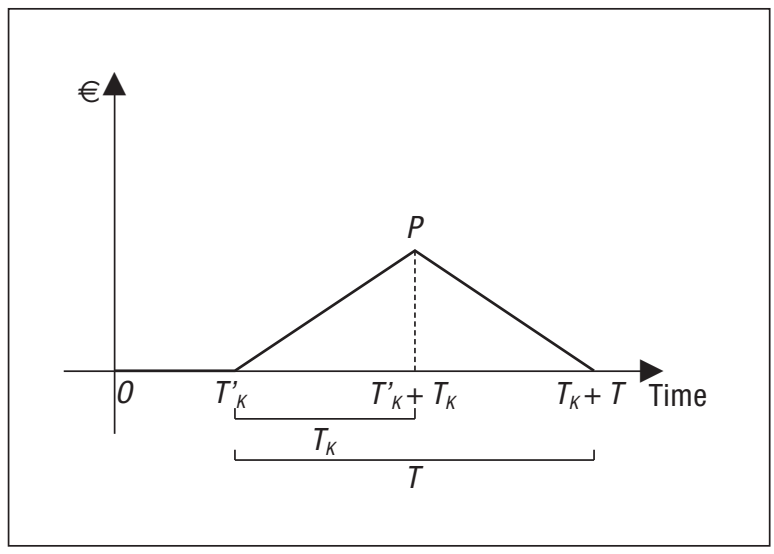

$P$ represents the maximum price at which the project can be sold once the patent has been marketed. So there exists an american type option to sell the project at a (reasonable) price function posed at the outset at an initial zero price fixed at the time when the patent development is underway, reaching a maximum $P T_{K}$ years after the patent is awarded. The «dry» value of the patent then is sought to drop to zero linearly.

At the research investment step, only a project abandonment option is considered.

Moreover, the option of a punctual investment in an industrial plant previous to the exploitation of the patent is also implemented.

\section{The valuation method}

A Monte Carlo simulation approach is undertaken, with the following assumptions:

I. The investment strategy follows a yes/no path: either we invest at the maximum rate or we stop investing.

2. Once the project is abandoned we leave it for good: no delay option is considered.

All processes are turned into discrete equations to carry on the simulations.

- Cost to completion:

$$
K(t+\Delta t)=K(t)-\mid \Delta t+\sigma(\mathbb{K})^{\frac{1}{2}} \sqrt{\Delta t \cdot \varepsilon_{\mid}}
$$


- Cash flow rates. A risk neutral modification of the real process is considered, so as the discrete diffusion equation is:

$$
C(t+\Delta t)=C(t) e^{\left(\alpha^{*}-\frac{1}{2} \phi^{2}\right) \Delta t+\phi \sqrt{\Delta t} \varepsilon_{2}}
$$

where $\varepsilon \mid$ and $\varepsilon 2$ are normally distributed $(0, I)$ with a correlation measured by $\rho$.

Time is divided into NT intervals of length $\Delta t=\frac{T}{N T}$.

The LSM (Least Squares Montecarlo) is implemented as follow:

Let be:

$W_{R}(i, j)=$ value of the discounted cash flows relative to path $i$ that are realized after the $j$ time step and without considering the existence of options.

$W_{0}(i, j)=$ value of the discounted cash flows relative to path $i$ that are realized after the $j$ time step, considering the existence of options.

$\hat{W}(i, j)=$ expected continuation value.

$-\mid \Delta t=$ differential cash flow at an investment stage.

$+C \Delta t=$ differential cash flow at the patent exploitation stage.

The optimal decision would be:

- Continue: if the expected continuation value is greater than the differential investment of the time step considered plus the sell/abandon value.

- Abandon/sell: if the expected continuation value is lower than the differential investment of the time step considered plus the sell/abandon value.

At the end of the life of the patent $j=N T W_{R}(i, N T)=$ $W_{0}(i, N T)=M \cdot C_{t=T}+C_{t=T} d t$ as we consider that the cash flows are realized at the end of the time intervals.

In order to estimate the expected continuation value we use LSM:
$\hat{W}(i, j)=\frac{1}{e^{\left(r_{f}+\lambda\right) \Delta t}} \bar{W}(i, j+l)$ at the investment stages.

$\hat{W}(i, j)=\frac{1}{e^{r_{f} \Delta t}} \bar{W}(i, j+l)$ at the exploitation stages.

Where $\bar{W}(i, j+I)$ is the least square estimate of the project value at $j+I$ time step. It is obtained fitting $W_{0}(i, j+1)$ to the state variables $K$ y $C$. We have used polynomials up to third power with cross terms as base functions at the investment stages $f(C, K)=a_{1}+$ $a_{2} C+a_{3} K+a_{4} C^{2}+a_{5} K^{2}+a_{6} C K+a_{7} C^{3}+a_{8} K^{3}+a_{9} C^{2}$ $+a_{10} C^{2} K$. At the exploitation step, as $K=0$, the following basis functions are used: $f(C)=a_{1}+a_{2} C+a_{3} C^{2}$ $+a_{4} C^{3}$.

We only contemplate the sell/abandonment option in the paths that are «in the money» in order to obtain an unbiased estimate of the expected continuation value. This happens when $W_{R}(i, j)-P<0$.

At the investment stages, if $\bar{W}(i, j)-l d t<P$ then the option is exercised and $W_{0}(i, j)=P$. If not, $W_{0}(i, j)=$ $\mathrm{e}^{-(r+\lambda) d t}$. $W_{0}(i, j+1)-1 d t$. If the investment in a industrial plant is taken into account Ip should be added at the time step previous to the exploitation stage. At this stage, if $W(i, j)-C d t<P$ then the option is exercised and $W_{0}(i, j)=P$. If not, $W_{0}(i, j)=e^{-r d t} \cdot W_{0}(i, j+1)$ $+C d t$.

This way we elaborate a Stopping Rule Matrix like this one:

\begin{tabular}{|c|c|c|c|}
\hline Path & $\mathbf{t = 1}$ & $\mathbf{t = 2}$ & $\mathbf{t = 3}$ \\
\hline 1 & Continue & Continue & Continue \\
\hline 2 & Abandon & - & - \\
\hline 3 & Continue & Continue & Abandon \\
\hline
\end{tabular}

Finally, the project value $W$ at time 0 is estimated discounting the cash flows of each path until an abandonment tag is reached and taking the average of all simulations.

\section{The tool}

A user friendly interface has been developed in order to help the valuator to carry on the process intuitively. 


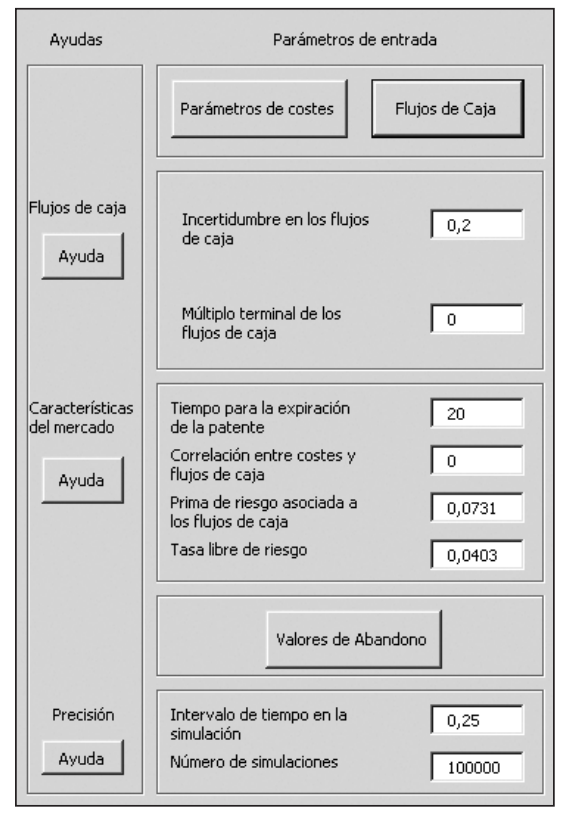

This is the parameter input screen where all the necessary variables for the model are set.

This is the results screen that includes:

- Classical NPV calculation (deterministic).

- Project value without options but with uncertainty.

- Project value with options and uncertainty.

- Average end investment time step.

- Average abandonment time step.

- Percentage of paths abandoned.

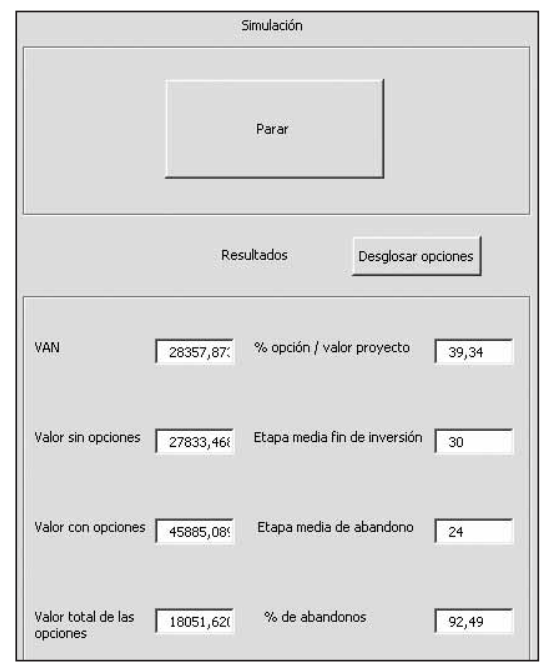

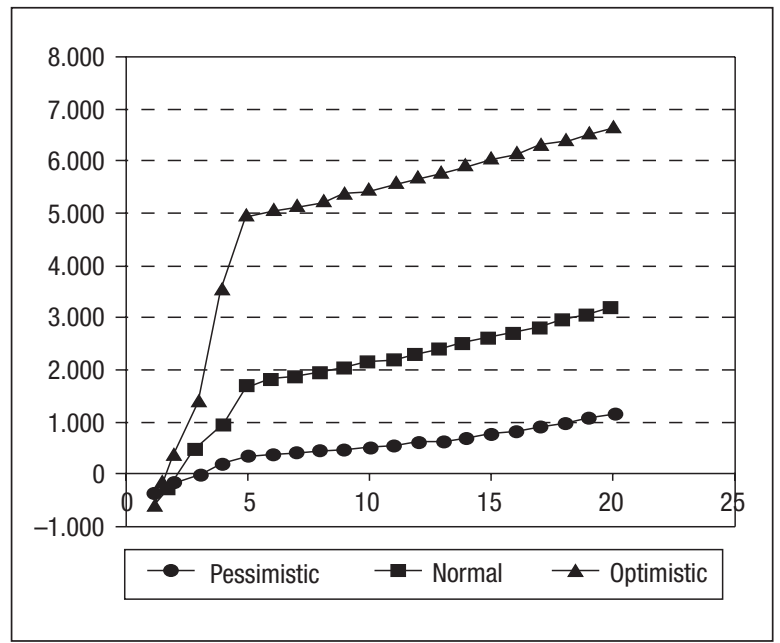

\section{Valuation of a patent related project}

As an application of the model, we show the case of a high tech company operating in the European market. This company has just finished with the research and development stages of their project and now they have been awarded with a patent so they will have 20 years for its exploitation. The company provided us with 3 possible cash flow scenarios (one pessimistic, one «normal» and one optimistic).

Considering a WACC of $15 \%$, an expected risk free interest rate of $5 \%$, a terminal value of $M=|0,0|$ and with an option profile starting at the classical NPV value and decreasing linearly to zero at the end of the life of the patent we have observed that the option adds a 52\% more value to the project.

It should be noted that the market value of the company is much closer to the real options value than to the NPV, proving the worthiness of the approach undertaken herein.

\section{References}

AMRAM, H., and KULATILAKA, N. ( 1999). «Real Options. Managing strategic investment in an uncertain world» Harvard Business School Press.

COPELAND, T., and ANTIKAROV, V. (200I). «Real Options, a practitioner's guide», Texere.

TRIGEORGIS, L. (1996). «Real options. Managerial flexibility and strategy in resource allocation».,MIT Press.

DIXIT, A. K., and PINDYCK, R. S. (1994). «lnvestment under uncertainty», Princeton University Press.

JÄCKEL, P. (2002). «Monte Carlo Methods in Finance», John Wiley\&Sons.

SCHWARTZ, E. (2004). «Patents and R\&D as Real Options» Economic Notes, vol. 33 ( I), pages 23-54,02. 